\section{Random Perturbations and Lattice Effects in Chaotic Population Dynamics}

Henson et al. (1) illustrated that populations consisting of integer numbers of individuals cannot be modeled as a continuum without paying attention to the discrepancy between the dynamics of discrete and continuous systems. The inverse of this problem (i.e., discrete models for continuous dynamical systems) has been well investigated since the work of Ulam (2), and several fundamental results in that area (35) can be applied to the questions raised by Henson et al.

One interesting issue is whether the refinement of the lattice (i.e., considering increasingly large habitat size) justifies, in the limit, using continuum models. As opposed to the ambiguous comments of Henson et al., we believe that there is no evidence at hand that discrete models converge in this sense. On the contrary, even on fine lattices, short cycles can be observed $(4,6)$. The discretized (lattice) Ricker model investigated by Henson et al. is no exception:
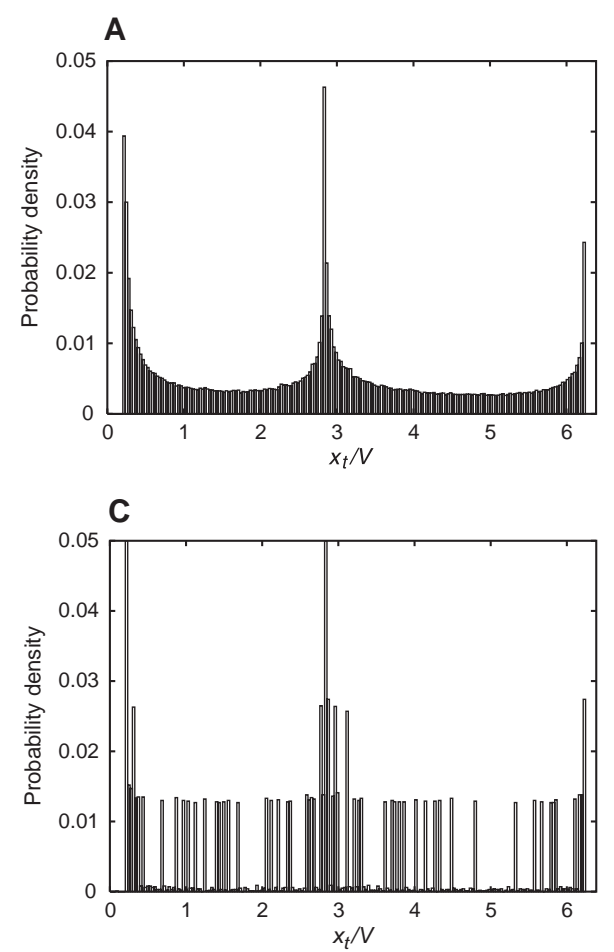

$$
x_{t+1}=F\left(x_{t}\right)=\operatorname{int}\left[b x_{t} \exp \left(-\frac{c}{V} x_{t}\right)\right]
$$

At different levels of discretization (i.e., differences in habitat size $V$ ), the model displays cyclic behavior. When $V=300$ [also shown in (1)], with initial condition $x_{0}$ $=1$, we observe a 59-cycle. Setting $V$ equal to 299 , however, results in an 8-cycle. Even radical refinement of the lattice can result in short cycles: an 8-cycle can be observed at $V=25,000, x_{0}=24$. In a discrete system, cycle length depends not only on lattice density (habitat size), but also on the initial condition. In the discrete Ricker model with $V=300$, shifting $x_{0}$ from 1 to 2 results in a jump from a 59 -cycle to an 8 -cycle.

Another question discussed by Henson et al. is to what extent the illustrated lattice effect remains perceptible if we add random noise. Recent results for expanding maps (6) indicate that extremely small ran-
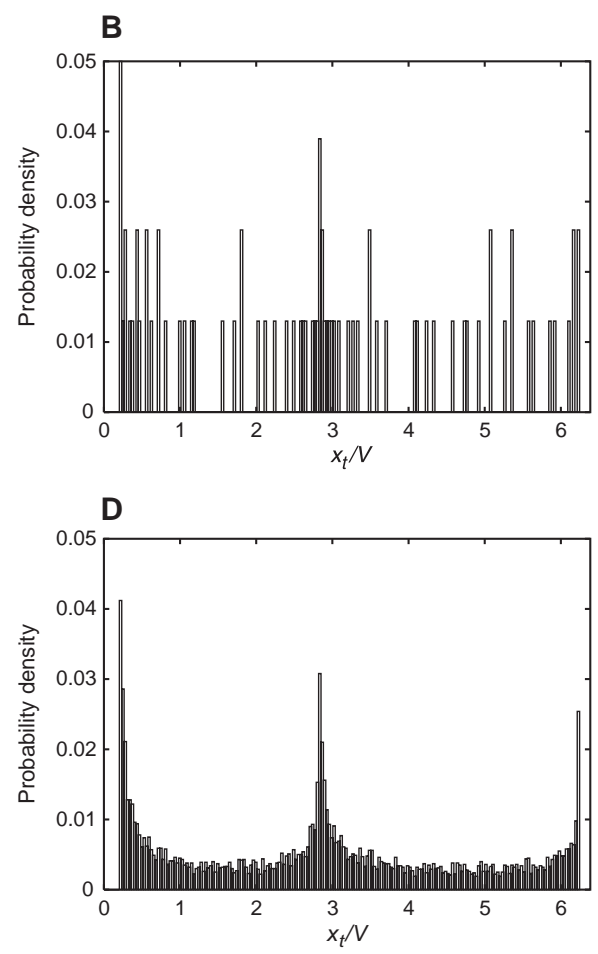

Fig. 1. Density function associated with the Ricker model, with $b=17, c=1$, and 10,000 time steps. Density function is averaged on 200 equal intervals. (A) Density associated with the continuous Ricker model with $V=300$ and $x_{0}=6$. (B) Density associated with the lattice Ricker model (Eq. 1) with $V=300$ and $x_{0}=6$; cycle length $=59$. (C) Density associated with the continuous Ricker model with $V=500$ and $x_{0}=2.19999$ on single-precision computer arithmetic; cycle length $=72$. (D) Density associated with the randomized discrete Ricker model (Eq. 2) with $V=300$ and $x_{0}=6$. dom perturbations destroy all lattice effects. Quantitatively speaking, the domain of the minimal random noise $R$ (resulting from birth fluctuations, for example) that is necessary to make the discrete system appear similar to the continuous one (in the sense that any statistical measurements on the population is predicted by the continuous model) is equal to the derivative of the map. In the case of the lattice Ricker model, this perturbation scheme can be written as

$$
x_{t+1}=\operatorname{int}\left[b x_{t} \exp \left(-\frac{c}{V} x_{t}\right)\right]+R_{t} \operatorname{sign}\left[D\left(x_{t}\right)\right]
$$

where $R_{t}$ is a uniformly distributed discrete random variable on $\left\{0,1,2, \ldots\left|D\left(x_{t}\right)\right|\right\}$, and $D\left(x_{t}\right)=F\left(x_{t}+1\right)-F\left(x_{t}\right)<b$ is the derivative of the lattice Ricker map (7). The "micro-perturbation" of Eq. 2 is illustrated in Fig. 1. Fig. 1A shows the invariant density function (statistical distribution of population size) associated with the continuous Ricker model. Fig. 1B illustrates the statistics of the lattice model; the dynamics are trapped in a 59-cycle and the statistics do not resemble those of Fig. 1A (8). Even radical refinement of the lattice does not provide satisfactory results. Fig. 1C displays the statistics obtained with single-precision "real"-type arithmetic on the continuous Ricker model with a 72-cycle. Fig. 1D shows the results obtained from the randomization in Eq. 2. Although Henson et al. applied a much larger random perturbation, they claimed that lattice effects are not completely destroyed, based on the observation that the randomized lattice Ricker model oscillates frequently around the fixed point. However, that observation has nothing to do with lattice effects, because the invariant density function of the continuous Ricker model has a steep maximum around the fixed point (Fig. 1A). The randomized model, even with our "minimal" noise, represents this correctly.

We conclude that lattice effects can be dominant even for very fine lattices (large populations) if random noise is disregarded. If random noise is included, lattice effects can still be important when looking at populations with very high instantaneous growth rates and very low random fluctuations in $R_{t}$. According to (6), the continuum approach is valid if $R_{t}>D\left(x_{\mathrm{t}}\right)$, which is often the case in real-life situations. Although we have demonstrated these phenomena on the Ricker model, the results in (3-6) indicate that similar behavior can be expected for a wide class of nonlinear maps, even in higher dimensions.

Gábor Domokos Cornell University

Department of Theoretical and Applied Mechanics

Ithaca, NY 14853-1503, USA 


\section{TECHNICALCOMMENTS}

István Scheuring

Eötvös University

Department of Plant Taxonomy and

Ecology

Research Group of Ecology and Theoretical Biology

H-1117 Budapest, Pázmány Péter sétány

$1 / c$, Hungary

E-mail:shieazsf@ludens.elte.hu

\section{References and Notes}

1. S. M. Henson et al., Science 294, 602 (2001).

2. S. M. Ulam, Modern Problems in Mathematics (Interscience, New York, 1960).

3. C. Liverani, Nonlinearity 14, 463 (2001).

4. Y. Kifer, Disc. Cont. Dyn. Syst. 3, 457 (1997).

5. M. Blanc, G. Keller, Nonlinearity 11, 1351 (1998).

6. G. Domokos, D. Szász, Disc. Cont. Dyn. Syst., in press.

7. Note that $R_{t}$ is not related to the size of the population. The maximal value of the growth rate is $b$, so a birth fluctuation of $b$ individuals is sufficient to produce the continuum effect.

8. In the discrete case, there are several invariant density functions; we have illustrated only one here.

\section{April 2002; accepted 12 August 2002}

Response: Domokos and Scheuring claim that, by adding a particular kind of noise to our discrete-state dynamical system, one obtains a good approximation of the invariant distribution of the continuous-state system. However, our models are not of the class for which they have proven this result (i.e., they are not "expanding maps"), and we believe that our aims differ from those of Domokos and Scheuring. Our interest is not in whether a special kind of noise, when added to discrete-state models, re-

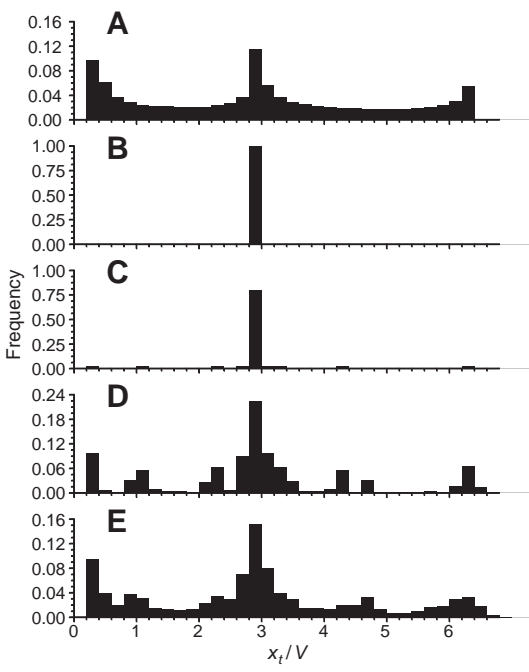

Fig. 1. Distributions of population densities from simulations of the Ricker model. Each distribution was created using a time series of $10^{6}$ data points after transients were discarded. (A) The distribution of the deterministic continuous-state Ricker model with $b=17$ and $c=1$. (B) Distribution of the deterministic lattice Ricker model (2) with the same parameter values as in (A). (C to E) Distributions of the stochastic lattice Ricker model with parameter values as in (A) and (C) $\sigma=0.001,(D)$ $\sigma=0.01$, and $(\mathrm{E}) \sigma=0.03$. sults in the recovery of statistical properties of the underlying continuous-state system. Rather, we find it interesting that several ecologically reasonable forms of stochasticity do not eliminate lattice effects arising from a biologically motivated model. Moreover, and most important from the scientific point of view, the presence of these lattice effects parsimoniously explains patterns we see in the experimental data. Domokos and Scheuring raise some issues that are interesting mathematically, but that do not bear directly on the scientific questions we addressed in (1).

Domokos and Scheuring have ignored our results for the LPA model (1), which applied directly to the experimental data, and instead have focused on our Ricker model example (2). They contend that "extremely small random perturbations destroy all lattice effects" and that our observation of lattice effects in the Ricker model illustration was based solely on the fact that the Ricker model oscillated frequently about the unstable fixed point. Fig. 1 shows, however, that these lattice effects are indeed real. Fig. 1A shows the invariant distribution of the deterministic continuous-state Ricker model, and Fig. 1B shows that of the deterministic lattice Ricker model for $V=$ 5. With a small amount of noise added (Fig. 1C), one can clearly see the significant departure from the continuous-state density of Fig. 1A in the direction of the lattice density of Fig. 1B. With larger amounts of noise, the departure is less pronounced but still evident (Fig. 1, D and E).

Typical time series simulations of the deterministic continuous-state and stochastic discrete-state Ricker models are shown in Fig. 2. The episodes of equilibration in the lattice map are much longer than those in the continuous-state map, and are therefore influenced by the lattice equilibrium. As $\sigma$ is increased to 0.03 (the value used in our paper), the situation continues to hold, although not to such a striking extent.
Fig. 2. Time series simulations of the $(\mathrm{A})$ deterministic continuous-state Ricker model with $b=17$, $c=1$, and initial condition $x_{0}=6$ and (B) the stochastic lattice Ricker model (2) with parameter values and initial condition as in (A) and $\sigma=$ 0.001 .
Another point raised by Domokos and Scheuring is that lattice cycles can have low periodicity even when the lattice mesh is extremely fine, and that it is therefore incorrect to assume that large population sizes render lattice effects unimportant. This observation is both correct and independent of the question of convergence "in the limit." We emphasized in our paper that the deterministic dynamics of the associated discrete-state and continuous-state models can be quite different, even for very large population sizes. As for convergence in the limit, Domokos and Scheuring are mistaken. It can be proven that, at least when the continuum attractors satisfy appropriate conditions of periodicity and hyperbolicity, "lattice attractors" will converge to the continuum attractor as the lattice spacing goes to zero. To our knowledge, Domokos and Scheuring are correct in asserting that such convergence has not yet been proven for the chaotic attractor of the Ricker map, nor has it yet been disproven.

Domokos and Scheuring are also correct in pointing out that the particular cycle on which the lattice system settles is often sensitive to the initial condition. When a discrete lattice is imposed on a chaotic attractor, the continuum attractor is, in general, broken up into multiple lattice cycles. A lattice system perturbed by noise can visit and revisit the various lattice cycles. It is this stochastic blending, together with noise-induced transient behavior, that recovers the signal of the continuum attractor. It is interesting to note that in this sense, stochasticity reveals the underlying deterministic signal instead of destroying it.

The issues raised by Domokos and Scheuring are interesting and, had they been made using the models we employed in (1), their relevance to our work might have been greater. Instead, to make their points, Domokos and Scheuring used a different model, much finer lattice mesh sizes $(V=300$ and $V=500)$, and a noise structure that appears to be inappropriate for ecological systems. One thing is certain
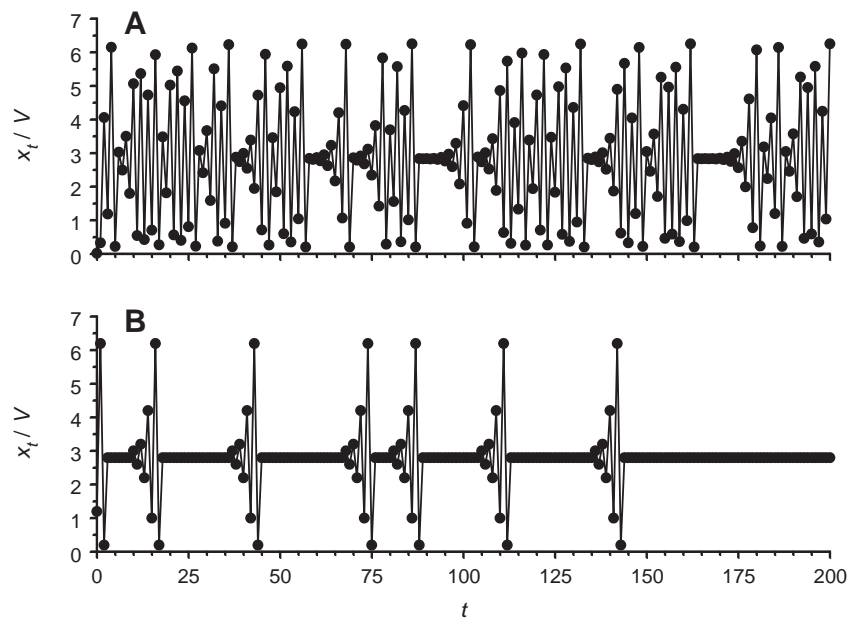


\section{TECHNICALCOMMENTS}

with regard to stochasticity and nonlinearity: The outcome of the mix can be both complicated and model-dependent. As a result, it is difficult to see how the remarks of Domokos and Scheuring bear on our original conclusions.

Aaron A. King

Department of Ecology and Evolutionary

Biology

University of Tennessee Knoxville, TN 37996, USA

Robert A. Desharnais Department of Biological Sciences California State University
Los Angeles, CA 90032, USA

E-mail:rdeshar@calstatela.edu

Shandelle M. Henson

Department of Mathematics Andrews University Berrien Springs, MI 49104, USA

R. F. Costantino

Department of Ecology and Evolutionary Biology University of Arizona Tucson, AZ 85721, USA

J. M. Cushing

Department of Mathematics, University of Arizona Tucson, AZ 85721, USA
Brian Dennis

Department of Fish and Wildlife Resources and Division of Statistics

University of Idaho Moscow, ID 83844, USA

References and Notes

1. S. M. Henson et al., Science 294, 602 (2001).

2. The stochastic lattice Ricker model we introduced for illustrative purposes is given by the map $x_{t+1}=\operatorname{int}\left[b x_{t} \exp \left(-\frac{c}{V} x_{t}+\sigma z_{t}\right)\right]$, where $z_{t}$ is distributed normally with mean zero and variance one.

5 June 2002; accepted 12 August 2002 